INPLASY

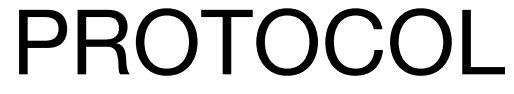

To cite: Luo et al. Adverse events associated with acupuncture therapy for psoriasis: a protocol for systematic review. Inplasy protocol 202040121. doi: 10.37766/inplasy2020.4.0121

Received: 20 April 2020

Published: 20 April 2020

Corresponding author: Ya Luo

dr.luoya2019@hotmail.com

Author Affiliation:

Guangzhou University of

Chinese Medicine

Support: STPGP

(2017B030314166)

Review Stage at time of this submission: The review has not yet started.

Conflicts of interest:

None.

\section{Adverse events associated with acupuncture therapy for psoriasis: protocol for systematic review}

Luo, Y1; Yu, JJ2; Yao, DN³; Xiang, CL4; Chen, WT5; Lu, CJ6.

Review question / Objective: Are there any potential risks of acupuncture therapy for psoriasis?What are the common adverse events associated with acupuncture therapy for psoriasis?

Condition being studied: In recent years, acupuncture therapy has been widely used in the treatment of psoriasis and is gaining growing popularity worldwide.However,with the increase of adverse events related to acupuncture therapy for psoriasis, its safety and risks are also of increasing concern.To the best of our knowledge,previous studies have focused on the benefits of acupuncture therapy in the treatment of psoriasis, whereas there is a lack of comprehensive assessment of its risks involved.

INPLASY registration number: This protocol was registered with the International Platform of Registered Systematic Review and Meta-Analysis Protocols (INPLASY) on 20 April 2020 and was last updated on 20 April 2020 (registration number INPLASY202040121).

\section{INTRODUCTION}

Review question / Objective: Are there any potential risks of acupuncture therapy for psoriasis? What are the common adverse events associated with acupuncture therapy for psoriasis?
Condition being studied: In recent years, acupuncture therapy has been widely used in the treatment of psoriasis and is gaining growing popularity worldwide. However, with the increase of adverse events related to acupuncture therapy for psoriasis, its safety and risks are also of increasing concern.To the best of our knowledge, previous studies have focused on the 
benefits of acupuncture therapy in the treatment of psoriasis,whereas there is a lack of comprehensive assessment of its risks involved.

\section{METHODS}

Participant or population: Patients with psoriasis.

Intervention: The interventions consisted only of acupuncture therapy, such as acupuncture, electro-acupuncture, fireneedle, moxibustion, cupping, auricular therapy, acupressure, acupoint injection, blood-letting, catgut embedding, etc.A single use or a combination of several acupuncture methods is acceptable.

\section{Comparator: Not applicable.}

Study designs to be included: Randomized Controlled Trials,Controlled Clinical Trials, cohort studies, cross-sectional studies, case-control, case-series, and case reports.

Eligibility criteria: Clinical studies that have reported adverse events associated with acupuncture therapy for psoriasis will be included.Non-human studies, reviews, author comments, expert opinions or consensuses, clinical guidelines, and duplicated studies will be excluded.

Information sources: Relevant studies will be searched in the following databases: PubMed, EMBAE, Scopus, ClinicalTrial.gov, the Cochrane Central Register of Controlled Trials (CENTRAL), China National Knowledge Infrastructure (CNKI), Chinese Biomedical Literature Database (CBM), Chinese Periodical Full-text Database (CQVIP), Chinese Academic Journal Database(Wan Fang Data). Besides, manual searches will be performed on the references of relevant studies for potentially eligible studies.

Main outcome(s): Any adverse events associated with acupuncture therapy for psoriasis, such as isomorphic response, Koebnerphenomenon, fainting, burns, hemorrhage, infection, allergy, swelling, blister, nerve or organ injury, pneumothorax, etc.

\section{Additional outcome(s): None.}

Data management: Two reviewers will independently screen the studies based on the inclusion and exclusion criteria, and then extract valuable information from all eligible included studies. Spreadsheets will be established to store the extracted information, such as study type, characteristics of participants, intervention measures, course of treatment, adverse events and the corresponding solutions, follow-up, etc.If there is any inconsistency, it shall be resolved by discussing or submitting to a third reviewer. For the information contained in the articles is incomplete, we will try to contact the author by phone or E-mail to supplement it.

Quality assessment / Risk of bias analysis: Given the possible variety of types of the included studies, the Cochrane Handbook Risk Of Bias assessment tool may not be applicable to all of them. Instead, the Risk of Bias of Individual Studies assessment tool and the McMaster University Harms scale (McHarm) both recommended by AHRQ(Agency for Healthcare Research and Quality) will be applied to assess the quality of different types of included studies.

Strategy of data synthesis: If adequate data is available, we will pool the incidence of adverse events of individual studies and then calculate the total incidence which have been weighted.The Metaprop package of stata 15.0 software will be applied for meta-analysis of proportions. Dichotomous data will be expressed as proportions with $95 \%$ confidence intervals (CI).Continuous data will be expressed in a descriptive way.The level of heterogeneity among the studies will be indicated by $\mathbf{l}$ value.If $12 \geq 50 \%$, it suggests that there's significant heterogeneity, and a random effect model will be applied. Otherwise,a fixed effect model will be applied. If the data is insufficient for quantitative synthesis, we will present a descriptive summary of the findings from the included 
studies, such as the common adverse events associated with acupuncture therapy for psoriasis, adverse events of different acupuncture methods, common causes of corresponding adverse events, etc.

Subgroup analysis: If feasible, subgroup analysis will be peformed according to different types of acupuncture methods and adverse events, treatment course, different types of psoriasis, etc.

Sensibility analysis: We will remove individual studies one by one and recalculate the pooled incidence of adverse events, and compare with the previous meta-analysis results,so as to evaluate the impact of each study on the pooled effect value and the stability of the results.

Language: There are no restrictions on language.

Country(ies) involved: China.

Keywords: Adverse events; acupuncture therapy; psoriasis; protocol; systematic review. 